\title{
Real-world challenges to collaborative text creation
}

\author{
Johannes Wilm \\ Fidus Writer \\ Flormansgatan $4 \mathrm{a}$ \\ 22354 Lund, Sweden \\ johannes@fiduswriter.org
}

\begin{abstract}
Academic real-time collaborative document editing faces a number of theoretical challenges. However, when implementing an editor in practice in the form of a web-based solution, a number of more practical challenges appear while some of the more theoretical problems seem less relevant. In this position paper we argue for working from a practice-based perspective when confronting issues of collaborative editing.
\end{abstract}

\section{Categories and Subject Descriptors}

H.5.3 [Information interfaces and presentation]: Group and Organization Interfaces - Web-based interaction, Collaborative computing, Computer-supported cooperative work, Synchronous interaction

\section{General Terms}

Performance, Design, Experimentation, Human Factors, Theory.

\section{Keywords}

collaborative editing, IT, web, contenteditable, user interface

\section{INTRODUCTION}

The process of creating texts, academic or otherwise, has always been social in nature, as our thoughts build upon those of others who came before us. In the world of academia, the various citation systems try to systematize the acknowledgement of outside influences on one's own writings, while listings of coauthorships on articles record the collaborative editing on a single document, at least theoretically. Until now this process was always one of individual academics writing at different times on the same document, sending it back and forth between the collaborators.

Only recently have we seen editors that allow for the collaborative editing of texts in real-time, and out of these Fidus Writer [1] is the only alternative known to us that is meant for nontechnical users in the humanities and social sciences [2]. There are LaTeX-based editors available as Authorea ${ }^{1}$ or writeLaTeX ${ }^{2}$ that require for the editor to write in a code language. It is our experience that within humanities and social

\footnotetext{
${ }^{1}$ https://www.authorea.com

2 https://www.writelatex.com
}

Permission to make digital or hard copies of part or all of this work for personal or classroom use is granted without fee provided that copies bear this notice and the full citation on the first page. For all other uses, contact the authors. Copyright is held by the authors.

DChanges '14, Sep 16-16, 2014, Fort Collins, CO, USA

ACM 978-1-4503-2964-4/14/09.

http://dx.doi.org/10.1145/2723147.2723154

\author{
Daniel Frebel \\ Fidus Writer \\ Linienstraße 154a \\ 10115 Berlin, Germany \\ daniel@fiduswriter.org
}

sciences that code-based editing is not an option for the users, as has also been the conclusion of other researchers [3]. We have spent the past two years programming as well as listening to the different types of users and potential users and what problems they would have with it. To develop an adequate word processor for all academic fields we decided to use a What You See Is What You Mean (WYSIWYM) interface using a reduced set of HTML elements and offering LaTeX as en export option (see Figure 1).

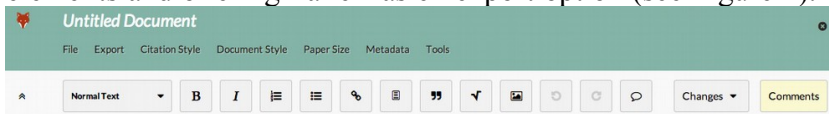

Figure 1: Fidus Writer only allows semantic editing - font sizes and types cannot be selected directly by the user.

\section{DATA SOURCES}

For this paper a number of data sources have been taken into account. Quantitative data comes from Fidus Writer's current 4300 beta users and their feedback through an online form accessible on all pages of the Fidus Writer application site August 2013 to September 2014, as well as a bug tracking system on Github ${ }^{3}$. Qualitative data comes from electronic interchange with less than 100 users who have had more extensive interchange with the Fidus Writer team through email and chat, after their initial form feedback. We have also had communications with other editing projects such as the teams behind Substance Composer ${ }^{4}$, CKeditor ${ }^{5}$, Aloha ${ }^{6}$, Booktype ${ }^{7}$, ShareLaTeX ${ }^{8}$ and writeLaTeX. Fidus Writer itself contains no system to analyze usage patterns and externally installed instances of Fidus Writer do not transmit any data back. The purpose of the data collection was at the time not part of a

\footnotetext{
${ }^{3}$ http://www.github.com

${ }^{4}$ http://www.substance.io

${ }^{5}$ http://ckeditor.com

${ }^{6}$ http://aloha-editor.org

${ }^{7}$ https://www.booktype.pro

${ }^{8}$ https://www.sharelatex.com
} 
scientific investigation but was done in order to set meaningful development goals for Fidus Writer.

\section{COLLABORATIVE EDITING AS A SECONDARY MODE}

We initially thought of editors writing collaborative constantly. We therefore believed that the editing process should take into consideration that several users would be connected to any document at all times. However, we discovered that our academic users mostly seem to edit their documents alone without the support of others. Users asked us to give them the option to write in the same document at the same time, but once it was available, they would seldom use it. It seems slightly more common to open the document at the same time, but only seldom will several users type simultaneously. Even though the part that handles concurrent edits initially was one of the most bug-ridden parts of the system, we have hardly received any error reports on technical users can use this feature easily. Figure 2 shows Fidus Writer used to collaborate between different users, whereby several forms of collaboration do not require realtime collaboration.

\section{DIFFING AND PATCHING}

Several projects have mentioned git and GitHub and that it would be a great idea to be able to use git and Github for finding changes to documents and then to patch them together [5]. This does not really seem to be practical for several reasons:

- Git is for programmers, most writers are not programmers. Especially in the humanities and social sciences users do depend on entirely graphical editors. And even a wellstyled diff output is too technical for them [3].

- Git is great to merge changes after edits of hours or days of work, not seconds. When writers need to merge their changes, it has to happen instantly and will usually only

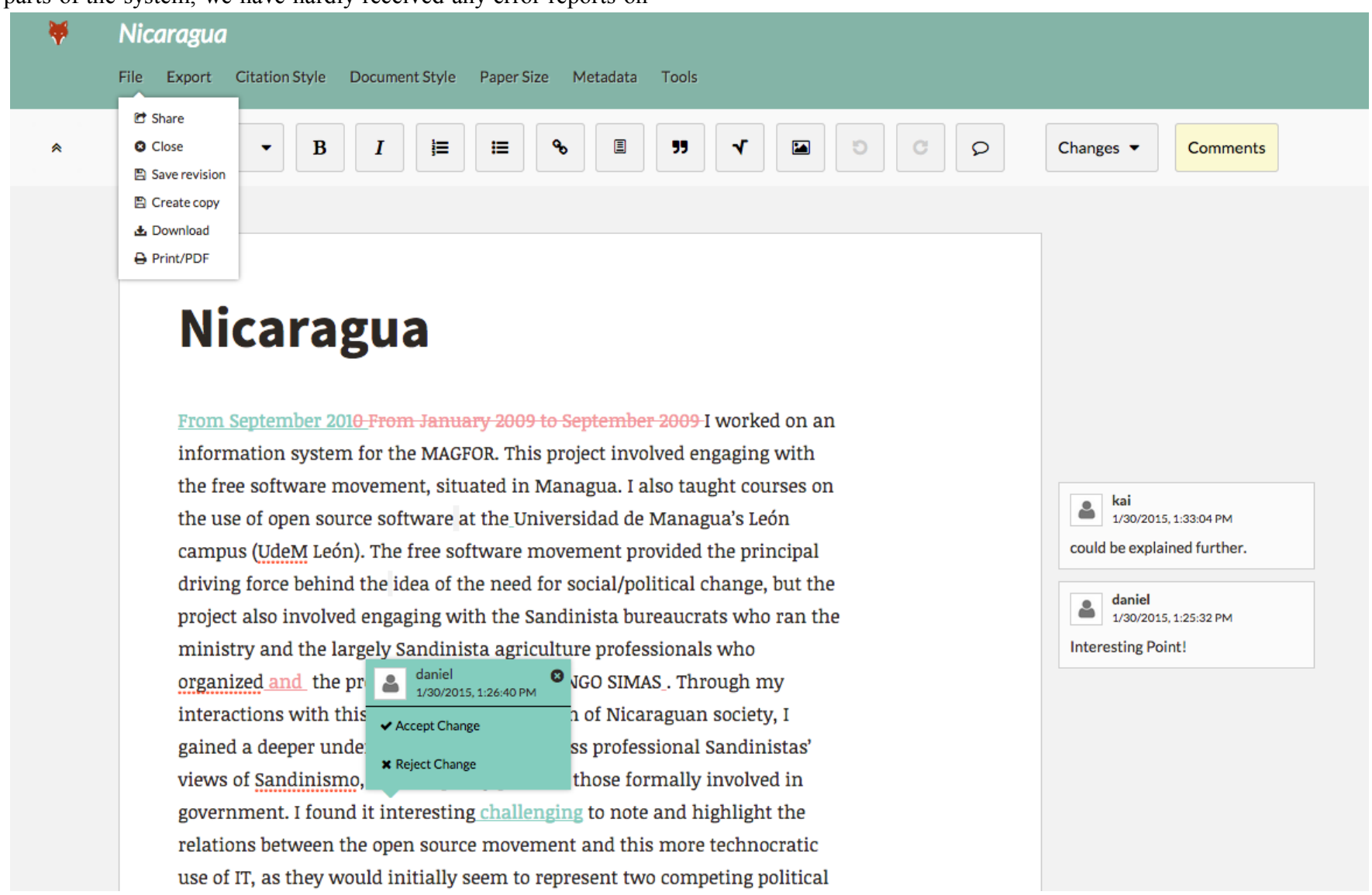

Figure 2: Fidus Writer allows collaborating using comments, tracked changes, revisions and simultaneous writing.

it. The writeLaTeX project has conducted tests of how often more than one user accessed the same document within one minute. The result was that only very seldom more than one user was in the same document [4].

Because handling collaborative editing is a lot more fragile and requires more resources on the server than working with a non-collaborative editor, we found out it made sense to have two editing modes: one non-collaborative, that is running most of the time, and a second, collaborative mode that runs when document edits are actually happening simultaneously. Changing between one mode and the other happens transparently, so that also non- concern edits of a few seconds and the change will therefore only contain a few changed letters. If the writer works for hours on a text and the Git server therefore records thousands of commits, the git history will be impractical to use.

- The default merging and diff display mechanisms for Git use single lines as the minimum unit of editing. For editors, a line is usually an entire paragraph. In programming it is not unusual to see lines of just 3-5 words. Writers of natural language documents cannot insert 
line breaks at will before finishing an entire paragraph. Only allowing edits from a single editor on an entire paragraph at any given time without creating merge conflicts would be rather restrictive. Git itself allows for using alternative merge mechanisms, that for example could use words rather than entire lines as the minimum unit. This would be an improvement, but Github does not allow this.

A git repository could nevertheless be used to store revisions of natural language document if these are in a format that can be converted without loss of information to a pure text document with a limited amount of characters per line.

\section{OVERLAPPING EDITS}

In theory, different editors could write at the exact same place in the document, and through the recoding of Operational Transformations (OTs) the editor can put these together to create a joint document of all editors [6,7]. In practice we found the problem to be somewhat different. For example, a text node would typically span just over a single word or up to a single line of text. If the one user was about to change "birthday" to "birthday celebration" while another user would delete the word "birthday" letter by letter, a combination after each user has hit two keys might look like "rthday ce." If two or more edits by different contributors concerned the same text node, one can be almost certain that diffing and patching the differences together will not result in an outcome that the writers will accept.

We found it to be more important that users would see the same text at the same time (as has also been argued by C.A. Ellis and S.J. Gibbs [6]), and therefore sufficient to use a Document Object Model (DOM) aware diff mechanism in the form of our diffDOM [8] library and applying diffs in consecutive order based on diff creating timestamp. These diffs can be undone and if more severe merge conflicts are encountered, all documents are reset to the current version of the first user who first opened the document. All this happens within a period up to a few seconds and mostly much faster than that, so that any potentially lost work is fairly limited.

Although our solution works well for us currently, in the future Fidus Writer will likely switch to a OT-based javascript solution using one of the common OT solutions such as TogetherJS ${ }^{9}$ or OT.js [9] so that we won't have to maintain our own library, but adjusting them in order to keep the current functionality. Adapting these will require some development efforts.

\section{UNDO}

Similar to overlapping edits, limitless undos could be handled by recording OTs for each collaborator while writing, and when executing the undo to either replay the creation of the document and just leaving the last OT of the current writer out, or by trying to undo the last OT of the current editor. In practice, replaying the entire chain of OTs from start to current point means keeping all these changes in memory which quickly grows too big to work with. Undoing just the last OT of the current user may oftentimes interfere with the edits of other users.

The solution in practice is therefore to allow a limited amount of undoing as long as the edits do not interfere with the edits of other users. In practice this means that as long as the last diffs produced by the user who is executing the undo command can be undone without causing merge conflicts, they are allowed to be undone. If one undo causes merge conflicts, it is assumed that it

\footnotetext{
${ }^{9}$ https://togetherjs.com
}

interferes too much with the edits of other users to be usefully undone and no earlier undos will be allowed to be undone.

\section{DOCUMENT HISTORY}

It has been a common suggestion to create a history bar that allows to go back to any moment in the creation of the current document by recoding all changes atomically. We tried something along these lines, but found it to be of little value because it consumed a lot of memory and users would not know how to handle the history bar as every change would be treated equally. In practice we found out it works much better if the writer has the ability to mark a certain current version as a historic document version to which the writer can later return. The current state of bibliography and images are recorded and saved along with the document in a zip file on the server which can be retrieved at a later stage.

\section{DOCUMENT OWNERSHIP}

All forms of production are social in nature, also textual productions. Fidus Writer beta users seem to want to collaborate on their text, but they want to do so in a controlled manner. We therefore have ended up allowing for the following types of user relations to documents inside Fidus Writer:

- document owner: can read, edit, turn tracked changes on/off, as well as give and revoke permissions

- collaborator: can read and edit

- reader: can read

A fourth mode has been requested repetitively, but we have not implemented it so far:

- commentator: can read and comment upon texts

\section{RECORDING OTS AS A REPLACEMENT FOR TRACKED CHANGES}

If one tracks each individual change a specific writer is responsible for, all the information needed to offer a graphical display of which editor is responsible for which part of the text the way the 'tracked changes' function in common word processors works, should theoretically be available.

In practice this information was not easily usable. Although the correct information was recorded, it's not accessible in the way one needs it. If the owner of a document wants to decide whether he wants to accept a certain part of a text written by another contributor, he generally wants to decide for all the text written by that user in the same line or paragraph, not just a letter or a word that happen to have been edited within a certain time range. Tracking changes is therefore something we do independently of looking at OTs.

\section{EDITING IN THE BROWSER}

It seems to be a common misconception that editing text in the browser is a stable feature. The corresponding attribute, contenteditable, turned out to be one of the least standardized and most error-prone features.

Issues encountered with contenteditable can be categorized in three categories: There are issues with the movement of the caret. The browsers allow the caret to move smoothly as long as it only moves within text content that is entirely editable. But if SVGs, Canvas-elements or multi-letter strings of text that are not editable individually, are descendants of the node with the contenteditable attribute, the caret will not always move correctly 
[10]. A second type of issue is the creation of HTML code that is counter-intuitive and not HTML5 compliant through the pressing of keys, for example when paragraphs are merged after typing the backspace key [11]. A third issue is related to the overall inconsistency in the HTML being output by the different browsers. In all of these cases the bugs differ slightly between browser, but each of the browsers holds a fair amount of bugs in each area.

Extensive work needs to be done using javascript libraries to work around the most common bugs in it. If one adds complex elements such as footnotes, citations or formulas, only a very limited number of projects exist that are interested in fixing it. Also, no usable library for combining contenteditable with collaborative editing existed until we started creating the diffDOM javascript library. If anything is stopping collaborative writing over the Internet, this is likely the area that most work should go into fixing.

The poor state of contenteditable in the browser implementations is likely a result of only a very limited amount of web developers working in the area of text editing [12]. A solution may therefore be to write an alternative to contenteditable entirely in javascript. This approach has successfully been employed by the CodeMirror project ${ }^{10}$, which is being used in different production environments. Although it can be used in a collaborative rich text editor, as demonstrated by the Firepad editor ${ }^{11}$, its primary focus is code editing and many adjustments would likely be needed to make it work with the more complex aspects of a collaborative academic editor. There are also several trials of creating rich text editors in pure javascript. Several of these are large in download and development team size and are not open source. The most prominent is Google Docs ${ }^{12}$. For niche purpose editors creating an editor like Google Docs does not seem financially viable. Other projects, such as the canvas-based Carota editor ${ }^{13}$ only serve as proof-of-concept and have not reached the production development stage. The closest open source, pure javascript, webbased editor made for richtext editing is Aloha 2.0 alpha. Should they be able to develop this into a working product with a plugin architecture that allows combining it with a realtime collaboration system such as that presently used by Fidus Writer, OT.js or similar, it may represent an approach or even be the library used by Fidus Writer and other similar editors as well in the near future.

\section{CONCLUSION}

Given the technical state of web technology in 2014, the creation of an open source collaborative academic web-based editor continues to be a difficult task. Some third party projects make it somewhat easier than two years ago, but it is still too complex for any single project team to resolve by itself. Additionally, as noted by C. Sun and C. Ellis [7], few people understand the complexity of realtime text editing and that this continues to be a field in need of further research. Fidus Writer has come close to the limits of what currently can be achieved, and has focused development efforts based on response from test users rather than to try to fix all theoretical problems with

\footnotetext{
${ }^{10}$ http://codemirror.net

${ }^{11} \mathrm{http}: / /$ www.firepad.io

${ }^{12} \mathrm{http}: / /$ docs.google.com

${ }^{13}$ http://earwicker.com/carota
}

collaborative text editing. However, it is still far from being an ideal editor. If we want to see such editors come in the next few years, it is essential that efforts are combined between the few projects that do exist in the field of webbased editing.

\section{ACKNOWLEDGMENTS}

Our thanks to the academic beta testers of Fidus Writers who have provided us with valuable feedback on use cases of collaborative academic editing as well as the teams of writeLatex, ShareLatex, CKeditor, Booktype, Substance.io and the Aloha Editor as well as the remaining members of the Fidus Writer team, Anne Wittorf Kojima and Takuto Kojima, for their input along the way.

\section{REFERENCES}

[1] Kojima, T.; Wilm, J. 2012-2014. Fidus Writer - an online collaborative editor made for academics. https://github.com/fiduswriter/fiduswriter

[2] Schöch, C. 2014. The right tool for the job: Five collaborative writing tools for academics. LSE Impact of Social Sciences Blog. (April 04, 2014). http://blogs.lse.ac.uk/impactofsocialsciences/2014/04/04/fiv e-collaborative-writing-tools-for-academics/.

[3] Munson, E. Keynote. DChanges 2013, September 10th, 2013, Florence, Italy. ceur-ws.org Volume 1008, http://ceurws.org/Vol-1008/keynote.pdf.

[4] Allen, J., Hammersley; J., Lees-Miller, J.; Oswald, H., Wilm, J. 2013. Moving scientific text production to the browser. Mozilla Festival 2013. (London, UK, October 2527, 2013).

[5] Lee, C. 2009. Gitpublish: Using Git and Restructured Text as a Publishing System. http://people.mbi.ucla.edu/leec/docs/gitpublish/intro.html.

[6] Ellis, C.A.; Gibbs, S.J. (1989). "Concurrency control in groupware systems". ACM SIGMOD Record 18 (2): 399 407. doi:10.1145/66926.

[7] Sun, C.; Ellis, C. (1998). "Operational transformation in real-time group editors: issues, algorithms, and achievements". "Proceedings of the 1998 ACM conference on Computer supported cooperative work". ACM Press New York, NY, USA. pp. 59-68.

[8] Kamermans, M.; Wilm, J. 2013-14. diffDOM - A JavaScript diffing algorithm for DOM elements. https://github.com/fiduswriter/diffDOM .

[9] Bauman, T. 2012-14. OT.js https://github.com/OperationalTransformation/ot.js .

[10] Wilm, J. 2013. Bug 873883 - Inconsistencies in cursor movement and deletion inside contenteditable element. https://bugzilla.mozilla.org/show_bug.cgi?id=873883.

[11] Koszuliński, P. 2013. Bug 114791 - Contenteditable issues related to backspace handling. https://bugs.webkit.org/show bug.cgi?id=114791 .

[12] Wilm, J. 2014. The World Wide West of Web Editing. http://fiduswriter.org/2014/09/14/the-world-wide-west-ofweb-editing. 